\title{
Communication et coopération ou le projet coopératif commutativement revisité
}

Henri Desroche

\section{(2) OpenEdition}

1 Journals

Édition électronique

URL : http://journals.openedition.org/communicationorganisation/1583

DOI : 10.4000/communicationorganisation. 1583

ISSN : $1775-3546$

Éditeur

Presses universitaires de Bordeaux

Édition imprimée

Date de publication : 1 novembre 1992

ISSN : 1168-5549

Référence électronique

Henri Desroche, "Communication et coopération ou le projet coopératif commutativement revisité », Communication et organisation [En ligne], 2 | 1992, mis en ligne le 26 mars 2012, consulté le 30 avril 2019. URL : http://journals.openedition.org/communicationorganisation/1583 ; DOI : 10.4000/ communicationorganisation. 1583

Ce document a été généré automatiquement le 30 avril 2019.

(c) Presses universitaires de Bordeaux 


\title{
Communication et coopération ou le projet coopératif commutativement revisité
}

\author{
Henri Desroche
}

\section{NOTE DE L'ÉDITEUR}

Tiré de Recma nº 18. pp. 87-1986.

1 S'agissant des problèmes de communication et d'organisation « communicationnelle »dans une structure coopérative et inter-coopérative mes observations participantes s'allongent maintenant au long d'un demi-siècle: depuis mes implications dans des communautés de travail, au début des années 1940, jusqu'à des explications contemporaines autour du 30ème Congrès mondial de l'ACI (Alliance coopérative internationale) à Tokyo en cette fin d'année 1992.

2 Peut-être sera-t-il compatible avec le présent dossier de cette revue que de prélever dans les miens trois contributions coopératives à une stratégie de communication qui serait :

- interactionnelle : ou la communication dans un carré d'acteurs

- inter-organisationnelle : ou la communication en modèle EMEREC

- intergénérationnelle : ou la communication dans une référence axiologique.

\section{Dans un carré d'acteurs : une communication interactionnelle}

3 C'est ce qu'une tradition de Congrès mondiaux aura nommé : la démocratie coopérative. Encore convient-il d'en circonstancier les modalités. 
Cette démocratie pose en effet un minimum de problèmes, lorsque la "communication " s'absorbe ou se résorbe dans une "communion » (selon la vieille distinction de Nicolas Berdiaeff), c'est-à-dire lorsqu'il s'agit d'une micro-société dont les membres se connaissent et se reconnaissent, se retrouvent et s'échangent fréquemment et spontanément, ont misé chacun une mise importante de l'enjeu collectif, maîtrisent à peu près leur responsabilité conjointe, décident généralement à l'unanimité et après mûres délibérations et disposent des services communs de gérances réduits au minimum. Je pense ici à cette coopérative ouvrière de Montevideo où le déjeuner dans l'atelier tenait lieu d'assemblée générale quotidienne, ou encore à cette coopérative de routiers pour lesquels une telle assemblée informelle se noue une ou plusieurs fois par semaine au cours d'une veillée amicale. Il en serait de même pour une petite société locale de consommateurs où le gérant est assisté quasi quotidiennement par la présence d'administrateurs ou de sociétaires bénévoles, ou encore pour un GAEC, une caisse locale de crédit mutuel ou même pour un mochav d'une cinquantaine de familles relativement homogènes.

Il n'en va plus de même lorsque, en vertu d'une simple mais impitoyable logique de croissance, la "petite» Kvoutza devient un "grand» Kibboutz et lorsque plus généralement les micro-sociétés se découplent et encourent simultanément la fierté de multiplier le nombre de leurs membres, l'euphorie de voir s'envoler les courbes du chiffre d'affaires, mais aussi les obligations de raffiner leur structure, de compliquer leurs communications internes, de recruter des compétences permanentes, d'embaucher une main-d'œuvre, de relayer leur démocratie directe dans une démocratie par procuration, de répercuter dans leurs rythmes les chocs en retour d'une gestion plus nerveuse, plus sociale, plus ciselée. C'est ainsi que peu à peu se dessine une structure type, que j'ai souvent imagée en la référant au quadrilatère coopératif dont ci-après un diagramme.

Diagramme 1 - La quadrilatère coopératif et ses clivages

\section{DIAGRAMME 1}

\section{Le quadrilatère coopératif et ses clivages}

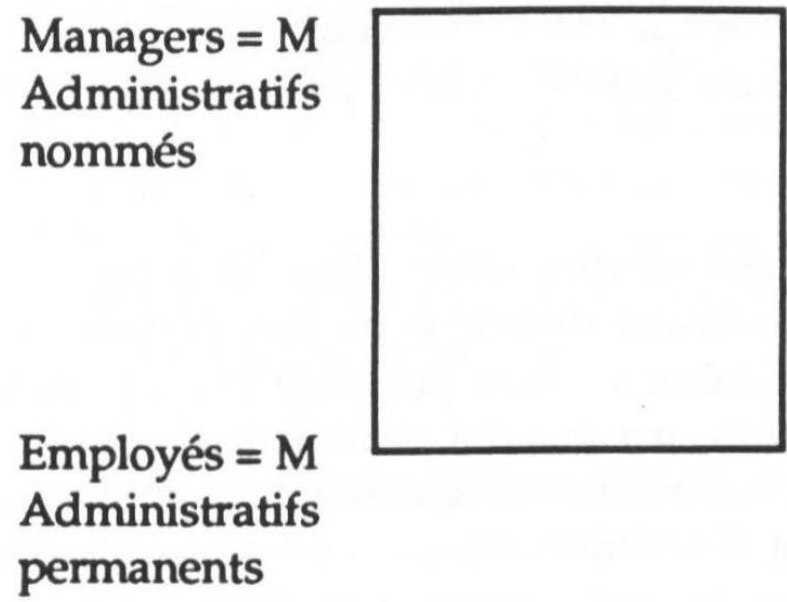

A = Administration
CA
Administratifs élus

S = Sociétaires

Administratifs souverains 


\section{DIAGRAMME 2}
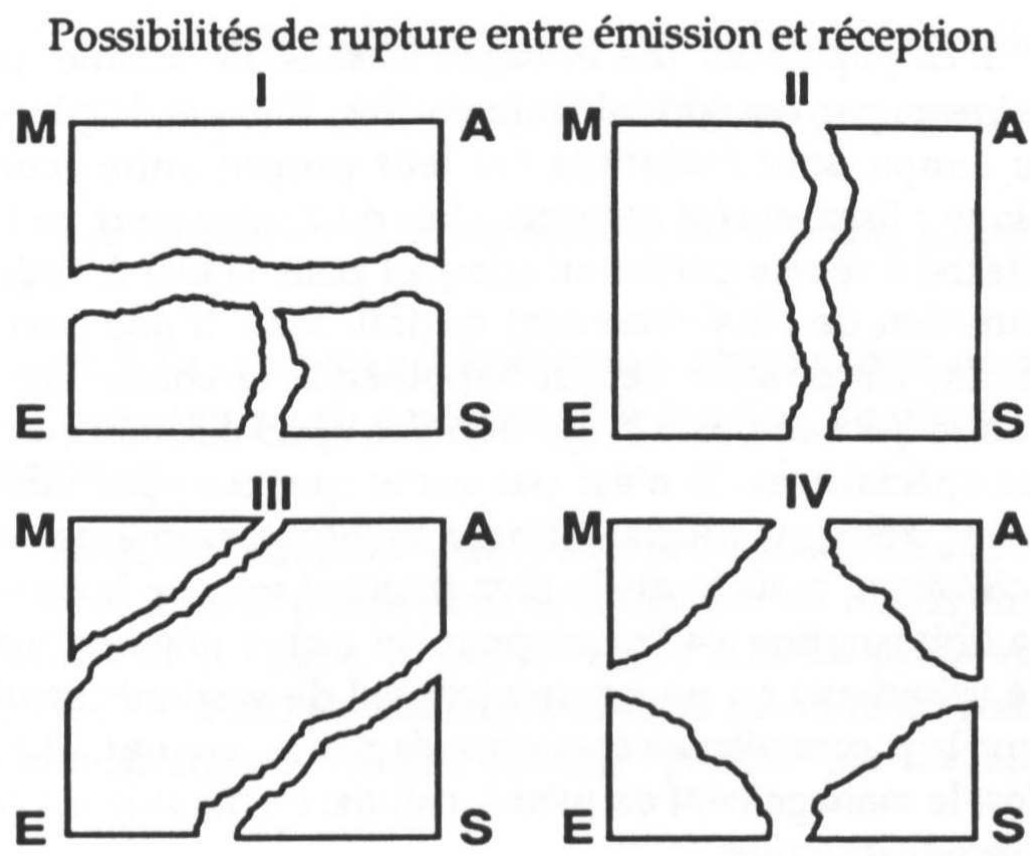
cette ou ces populations qui sont touchées par ce label. Et l'on s'aperçoit que ces populations sont généralement ${ }^{1}$ au nombre de quatre :

7 1. La population des sociétaires proprement dite, c'est-à-dire des membres ayant acquitté leur part sociale et participant, pour une dose significative, à des transactions régulières entre leur ménage ou leur firme et la centralité de l'établissement coopératif. adopté pour la prise de décision.

2. La population des administrateurs élus. Très rapidement en effet la démocratie directe n'est pas praticable. Serait impensable, onéreux s'il était pensable et inopérant s'il n'était pas onéreux, un schéma dans lequel tous se mêleraient de tout. L'assemblée délègue donc ses pouvoirs à un conseil restreint d'administration, les administrateurs étant élus en tant qu'ils sont perçus, à un titre ou à un autre, comme représentatifs, et pour des durées variables selon les cas, y compris les cas de révocabilité.

3. La population des managers nommés. Et nommés précisément par ces administrateurs élus. Ceux-ci, la plupart du temps, sont mobilisés sur leur propre unité économique : firme ou/et ménage. L'un ou l'autre peut en être détaché à temps partiel ou complet pour veiller à l'administration de l'établissement central. Mais d'une part ce fait est temporaire, ce qui hypothèque la continuité, et d'autre part ce poste exige déjà des spécialisations, donc des spécialistes. Il n'est pas exclu que ces spécialistes soient devenus ou deviennent bientôt eux-mêmes des sociétaires ; mais le cas le plus fréquent est que le conseil d'administration s'adjoigne pour ces tâches une personnalité (directeur) ou un groupe (conseil de gestion) recrutés pour leur compétence et contractés par un contrat ad hoc. C'est le management ou même, comme on dit aujourd'hui, la technostructure. 
11 4. La population enfin des employés salariés. Dans la mesure où l'établissement rentrai, pour soutenir le dynamisme de l'agrégat, est amené à s'étoffer, il ne saurait se suffire d'une personnalité ou même d'une équipe de pure conception. Ses bureaux se multiplient pour assumer les tâches comptables, commerciales et financières. Il est amené à postuler des installations d'entreposage, de conservation, de conditionnement, voire de transformation. Il doit prévoir des communications de marchandises, de personnel, d'informations, donc s'équiper d'un parc de véhicules et de dispositifs de transmission; enfin ledit établissement ne peut échapper à l'obligation d'étendre son emprise en aval ou en amont de la transaction fondamentale initialement requise par ses mandants. Bref, son dispositif de conception est - pour assurer leur fonctionnement d'embaucher une maind'œuvre en veston ou en salopette et de s'assurer son emploi par un contrat qui est ordinairement un contrat de salaire.

Ainsi de campent peu à peu les quatre pôles du quadrilatère :

$\mathrm{S}:$ Sociétaires = agréés par l'assemblée générale

A : Administrateurs = élus par les sociétaires agréés

M. : Managers = contractés par les administrateurs élus

$\mathrm{E}:$ Employés = salariés par les managers contractés

Pour compléter le commentaire du schéma, ajoutons que :

- en $\mathrm{S}$, l'assemblée générale trop vaste peut être amenée à se démultiplier en assemblées de localité, de district ou de section,

- en $\mathrm{A}$, des administrateurs élus peuvent également être détachés à plein temps pour se conjuguer au management permanent et même, la clef de voûte sera un personnage (le PDG)

à la fois élu comme tout autre membre du conseil d'administration et reconnu comme " patron » par les managers eux-mêmes,

- en $\mathrm{M}$, les managers peuvent être recrutés parmi des sociétaires, mais le plus souvent, n'étant pas eux-mêmes coopérateurs, ils auraient tendance, à se centrer plutôt sur le développement de l'établissement, c'est-à-dire de la société, que sur la satisfaction des sociétaires, de leurs ménages ou de leurs firmes,

- en E, enfin, les salariés auront plutôt tendance à faire passer les intérêts syndicaux de leur position professionnelle avant leur intégration à la société coopérative, même si par ailleurs ils cumulent leur fonction de salarié avec une position de sociétaire dans la même coopérative.

Le pont aux ânes de la démocratie coopérative est précisément d'obtenir des communications, voire des convergences, entre ces quatre pôles. On sait maintenant qu'un tel fonctionnement ne va pas de soi.

Entre S et A - la communication est en principe garantie par l'élection. Mais, surtout dans les grandes coopératives, cette élection n'intervient qu'au deuxième ou troisième degré, c'est à dire que la désignation se fait lointaine, formelle, filtrée par l'importance éventuelle des groupes de pression.

Entre $\mathrm{A}$ et $\mathrm{M}-\mathrm{M}$ est en principe un pouvoir exécutif, tandis que A exerce un pouvoir législatif. Mais on sait combien les entreprises sont susceptibles de se renverser, dans ce type de rapports. La force de l'exécutif lui vient de sa permanence, de sa familiarité avec les dossiers, de ses compétences requises, d'un training spécifique, du temps dont il dispose pour peser les décisions à prendre, puisqu'il est payé pour prendre tout son temps à les préparer et à les mûrir. Toutes conditions qui risquent de faire défaut à $\mathrm{A}$. 
21 Entre $\mathrm{M}$ et $\mathrm{E}$ - étant donné le régime salarial actuellement prévalant pour cette partie du schéma, les relations risquent de n'être guère différentes de ce qu'elles sont un peu partout entre employeurs et employés, intérêt d'une entreprise et intérêts de ses salariés, compris avec contestation ou radicalisation des seconds à l'endroit et à rencontre de la première.

22 Entre E et S - enfin. Si on va de proche en proche et d'instance en instance juridiquement et formellement : $\mathrm{S}$ est le patron de $\mathrm{E}$, puisque $\mathrm{S}$ a choisi A qui nomme $\mathrm{M}$, qui a embauché E. En fait, c'est plutôt le contraire qui se produirait : certes, E sert et dessert S, mais les filières à remonter par $S$ sont et trop longues et trop complexes et trop aléatoires pour que, sauf exception, il ne se contente pas du service tel qu'il lui est livré.

On pourrait ajouter à cette liste d'autres redistributions paradoxales: les diagrammes proposés figurent seulement les plus importants des clivages ou/et des coalitions ${ }^{2}$.

\section{En modèle « EMEREC » : une communication interorganisationnelle}

EMEREC, c'est-à-dire en régime d'EMissions et RECeptions réciproques, ou non, interorganisationnelles, c'est-à-dire entre types d'organisations, et plus particulièrement dans le cas d'une structure coopérative: entre son organisation comme entreprise et son organisation comme association, ou plus généralement son organisation en termes d'appareils et son organisation en termes de réseaux.

Un tel inter-inter peut fonctionner (ou dysfonctionner)

- soit à l'intérieur d'une entreprise : soit dans le sens vertical (appareils $\mathrm{M}+\mathrm{E}$ versus réseaux $\mathrm{S}$ +A) soit dans le sens horizontal (sommets M+A versus bases E+S), cf supra diagramme II.

- soit dans un agrégat interentreprises (fédération, confédération, coordination, complexes etc) entre les composantes et leur surordination.

- soit dans les corrélations, interrelations, conventions, régulations, intervenues ou à intervenir entre : un ensemble ONG (Organisation Non Gouvernementale) et un esemble étatico-administratif comme ensemble OG (Organisation Gouvernementale)

- soit dans les dispositifs précédents déployés ou redéployés toujours comme autant de couplage (ou de découplages) entre des appareils et des réseaux mais en termes de corrélat Nord-Sud (OG + ONG NORD versus ONG + OG SUD).

$\mathrm{Au}$ terme de cette longue et sinueuse entrée en scène, un concept pourrait éclairer ses méandres : celui d'animation, et en l'occurrence d'animation coopérative. Il s'est surtout affiné dans des chantiers coopératifs de pays sous-développés, en raison même de la distance qui séparait les appareils de développement et les réseaux villageois, et en vertu des requêtes d'une planification participante et participée. Il a été réinventé pour figurer en place honorable dans le VIe Plan français, compte tenu que, par ailleurs, son emploi tous azimuts et à toutes les sauces ne rend pas son élucidation aisée. Dans le contexte africain, l'instruction circulaire de Mamadou Dia est sans doute le document anticipateur le plus clair, le plus topique.

Si on veut généraliser, sans s'envoler dans le lyrisme gratuit ou s'enliser dans une critique pisse-vinaigre, peut-être le mieux est-il de se référer à un système d'émission-réception greffé sur le quadrilatère dont la démocratie coopérative offre les éléments. La difficulté sera que ce schéma s'applique à de multiples rapports : entre l'établissement central et les 
unités périphériques, entre des organisations « faittières » et des organisations de bas, ou même entre la « direction » et les « membres », pour parler le langage des rapports ACI.

Pour ramener les variables de tous ces cas à l'unité d'un prototype, je dirais volontiers que l'animation, d'une part, se définit comme un système de communication que, d'autre part, il s'agit de communication entre des appareils et des réseaux et qu'enfin cette communication se diversifie selon un double code: un code d'émission, un code de réception. Le problème final de cette animation dans une démocratie coopérative se réduit à sa capacité de doter ou non appareils ou/et réseaux en présence d'un équipement émetteur et d'un équipement récepteur.

\section{Diagramme 3 - Animations \& communications}

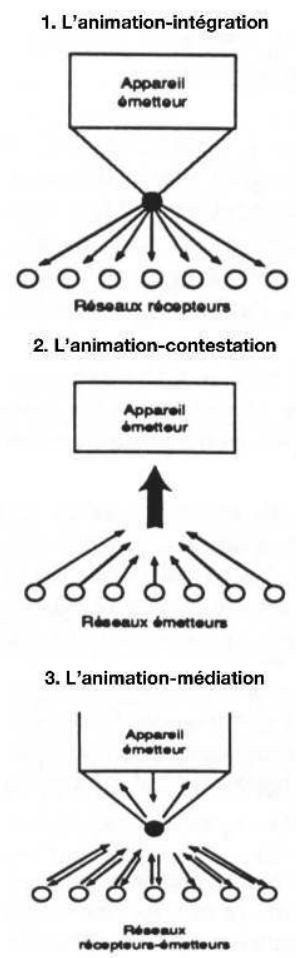

Lorsque chacun des partenaires dispose de son poste émetteur-récepteur, c'est à eux de jouer, le rôle de l'animation n'étant guère plus, dès lors, que de parer aux brouillages et de veiller à la qualité du son. Les diagrammes ci-avant évoquent dans cette perspective les trois cas majeurs d'animation. Pour les désigner, je prends à dessein des termes axiologiquement neutres, mais il est évident qu'une idéologie pour ou contre l'une ou l'autre d'entre elles peut aisément nourrir soit une sublimation, soit une caricature.

Cas 1. L'animation-intégration. Le pouvoir des appareils a déterminé une politique et pris des décisions à partir de ses laboratoires. Mais pour en assurer l'application il convient d'obtenir la participation des réseaux. Il s'agit de leur vendre cette politique ou cette décision et il s'agit qu'ils l'achètent. La pédagogie en est délibérément et consciemment directive. Quelle que soit l'axiologie ou la méthodologie, la participation offerte consiste à occuper toute la part, et seulement la part, qui aura été préalablement dévolue. Les appareils ont un poste émetteur sans poste récepteur, et les réseaux sont dotés d'une poste récepteur sans poste émetteur. 
31 Cette animation s'étire de l'explication de vulgarisation à l'incantation d'embrigadement. Elle va de tout ce qu'il y a de plus plausible dans la diffusion d'acquis scientifiques à ce qu'il y a de plus suspect dans le lavage de cerveau. Elle peut pousser sur la persuasion comme sur l'intimidation. Opérer un ralliement ou dissimuler des annexions. Provoquer les intelligences à une réflexion ou s'assujettir des réflexes conditionnés. Servir l'intérêt général ou asservir à une tyrannie. Cuvrer pour une intégralité organique ou fomenter un intégrisme totalitaire. Car l'animation d'intégration peut tomber dans l'intégration de manipulation.

Cas 2. L'animation-contestation. Inversement au cas précédent, ce sont les réseaux qui émettent et qui demandent essentiellement aux appareils de recevoir leur message. Ce message peut-être une doléance, une objurgation, une protestation, un sursaut d'impatience ou de frustration, la demande d'une trêve ou la menace d'une grève, à la limite la revendication d'un renversement de rôles ou d'un déplacement des centres de décisions. Son ressort est non pas la clarté d'un savoir, mais la force d'un vouloir, la latence d'un pouvoir et même parfois l'émergence d'un contre-pouvoir.

Il serait facile d'aligner les versions sublimantes: c'est de ce côté que se tiennent la spontanéité créatrice des masses, les presciences des prophétismes sociaux, les intuitions des utopistes, les militances populaires, l'objection de conscience, les marginalismes féconds, les radicalismes purs et durs, le non aux tentations de compromis, une société en train de se faire contre des sociétés par trop toutes faites. Il ne serait pas impossible non plus, bien que ce soit à contre-courant, de déceler les versions de ce contestationnisme quand d'aventure il succombe à ses démesures : ce qu'il draine de grandiloquence ou de mégalomanie, ce qu'il comporte de déviance ou d'aberration, ce qu'il implique d'allergies et d'intolérances... Car l'animation-contestation peut simplement panacher les démesures d'un cabotinage.

Cas 3. L'animation-médiation. Ce serait le cas où les partenaires en présence disposent chacun et d'un émetteur pour livrer leurs messages et d'un récepteur pour capter les messages d'en face. L'animation est l'agence d'installation de ce dispositif réciproque et qui en assurerait le service après vente. Son rôle est dès lors purement maïeutique : acheminer des transmissions en s'assurant de leurs termes pour que de tels messages soient effectivement reçus et que le dialogue se noue. Cet office est effectivement analogue à celui d'un médiateur, d'un interprète en traduction simultanée et réciproque, voire d'un ombudsman, encore que l'animation ne dispose ni des pouvoirs ni du prestige de ce personnage. Tout au plus peut-elle suggérer et susciter un tel arbitrage. Ce n'est pas elle qui négocie : elle négocie la négociation.

Ce profil a de quoi retenir les préférences de tous ceux dont le zèle s'active à promouvoir une planification démocratique. L'ACI a recommandé : «Direction et responsables (les appareils) doivent à tous les niveaux être en contact étroit avec l'opinion des membres individuels (les réseaux), et la machine doit être mise en place pour transmettre les recommandations de "la base" au haut de l'échelon (from the "grass roots" upward) ${ }^{3}$. C'est relativement facile à dire. Il ne s'ensuit pas que cette forme d'animation ne puisse être elle-même piégée par le formalisme, le double jeu des cadences atermoyantes, des évaporations pusillanimes, des partis pris subreptices ou des procédures machiavéliques.

C'est ainsi que l'animation en général - et l'animation coopérative en particulier - se trouve être dans le cas de la langue d'Esope, prometteuse de tous biens et chargée de tous maux. Et cela quelle que soit sa forme. Mais, hic et nunc, il y a une forme préférable à 
d'autres, surtout si celle qui est préférée ne tombe pas dans sa caricature. En un sens, il est un problème de l'animation coopérative qui demeure une clef de la démocratie coopérative. C'est l'un de ces métiers nouveaux dont Charles Gide annonçait l'émergence concomitante à la récession de "cette forme du patronage qui s'appelle la direction ». Selon lui, en effet, une autre forme " acquerra de plus en plus d'importance économique au fur et à mesure que la substitution de l'entreprise collective à l'entreprise individuelle groupera de plus grandes masses d'hommes. Savoir «faire faire » aux autres deviendra une condition de succès encore plus efficace que savoir faire soi-même $»^{4}$.

Du coup et du même coup, si la "démocratie coopérative » en était à conjuguer une double communication optimale selon le carré des acteurs, d'une part et, d'autre part, selon un modèle EMEREC, son régime « communicationnel » aurait anticipé et rejoindrait à moins qu'il ne soit rejoint ou même devancé par ce qu'un projet d'entreprise réclame d'une éthique, d'une culture ou même d'une mémoire d'entreprise en termes d'«excellence» ou/et de qualité, de troisième cycle ou de cinquième pouvoir, d'intelligence, de gestion et de mobilisation des ressources humaines ${ }^{5}$.

\section{Dans une référence Axiologique une communication intergénérationnelle}

Axiologie? Retenons ce terme de référence pour désigner ce que la conjoncture fait valser autour des éthiques - économique et entrepreneuriale - et ce qu'une tradition coopérative aurait voulu définir en termes de "principes » ou même de " valeurs ", avant de distinguer, dans ces références, ce qui relève d'une constante stratégique et ce qui appartient à des variables jurisprudentielles...

Cette tradition coopérative voudrait remonter à des «principes » dits « rochdaliens », du non de Rochdale, la petite agglomération anglaise qui, au temps d'un capitalisme "manchestérien", aurait élaboré le prototype devenu archétype des sociétés coopératives de l'Europe, de l'Occident, et finalement d'une expansion Nord-Nord puis Nord-Sud... Mais c'est seulement un demi-siècle plus tard, dans la première moitié des années 1890, que la pratique de ces "principes» aura été plus théoriquement interrogée...

Cette interrogation s'est ensuite réitérée à plusieurs reprises :

- dans la décennie des années 1930, moyennant une double enquête archivistique et conjoncturelle sur le nombre, le discernement, la définition et l'obligation des principes " coopératifs » assimilés à des principes « rochdaliens".

- dans la cascade de trois congrès : Vienne 1966, Hambourg 1969, Varsovie 1972 pour une redéfinition des susdits principes comme facteurs d'une démocratie coopérative. Ces discussions ou promulgations ont été connotées ailleurs.

- enfin dans les réitérations des trois derniers congrès de la même ACI : à Moscou sur les « horizons ", à Hambourg sur les « finalités », à Stockholm sur les « valeurs » du développement coopératif international. Sur la lancée, une commission « indépendante » s'est même évertuée autour d'une « axiologie » de sa formulation ou même... sa formalisation. Question: cette axiologie transmise et retransmise, considérée et reconsidérée intergénérationnellement, livre-t-elle des messages en termes de « communications »?

Réponse : oui et non. Ou plutôt : un message latent et quelques messages manifestes. 
Le message latent serait lui-même double: celui d'une généalogie, celui d'un apparentement.

Une généalogie : à partir de matrices associatives-communautaires attestées et parfois testées dans des utopies pratiquantes. Même le prototype de Rochdale se réfère, dans sa Loi Première (First Law), à une telle matrice : celle des communautés owénites, pour un " nouveau monde moral », annoncé par la " religion rationnelle », d'un millenium " mis en pratique » (millenium in practice). Il s'agit pour ce mouvement social de ses «souvenirs d'enfance »... Souvenirs refoulés, mais périodiquement ressurgis dans des expériences précaires ou durables, celles des coopératives communautaires multifonctionnelles (kibboutzim, communautés de travail, villagisations à qualité de vie... ) ; celles aussi des CBM (coopératives à buts multiples) proliférantes dans le Sud-Est Asiatique, selon enquête du BIT. Il va de soi que dans de tels modèles coopératifs les problèmes de communication obtiennent plus spontanément des solutions de « communion » : la passion d'une convivialité nourrit l'action coopérative.

Faute de quoi, le développement unilatéral (et unifonctionnel) des entreprises coopératives se cherche - communicationnellement parlant - suppléance, rattrapage, rescousse soit dans une inter-coopération, soit même dans l'intégration de celle-ci à une globalité d'économie sociale. Les problèmes de communication y deviennent des exercices de concertation pour ne pas dire de conversation, plus rarement de cooptation ou de corroboration.

Par contre, des messages manifestes se laissent épingler aux deux bouts de la chaîne, c'est-à-dire du centenaire 1892-1992. D'où la connotation qui suit.

Cette année 1992 sera pour l'Alliance Coopérative Internationale l'année d'un trentième Congrès mondial au Japon. Congrès géographiquement notable : pour la première fois, cette ACI prend le grand large et son congrès quitte l'Europe où depuis 1895 on s'était prudemment calfeutré. Et historiquement non moins significatif, puisque, fût ce sans le savoir, en ponctuant 1992, ce Congrès ponctue un centenaire sur, sinon l'histoire, du moins la préhistoire de l'ACI, en réitérant - contenant et contenu - une plateforme de principes ou même de valeurs qui, depuis lors et au long du long d'une histoire bientôt centenaire elle-même en 1995, avaient été tenus dans l'oubliette ou refoulés dans les cryptes d'une inhibition... Ce trentième Congrès, de 1992 et au Japon, serait-il celui où se défouleront des souvenirs d'enfance...?

Le contenant : parce que, en ce temps là, en juin 1892, et moyennant une Entente cordiale franco-britannique, se tenait à Rochdale ce qui pouvait être considéré comme un essai décisif pour lancer une Alliance coopérative internationale sous forme d'une «International Alliance of the Friends of Co-opérative Production ».

Le contenu : parce que le Proposai, alias la Proposition, et son Adresse Inaugurale, prenait la forme d'un Manifeste pour postuler entre autres :

- la contestation d'une hégémonie : celle qui s'exerçait au bénéfice des coopératives de consommation et de leur édification économique dominante ;

- la requête d'une intégration des producteurs, soit au titre de leur coopérative spécifique, soit au titre de leur sociétariat ou de leur participation, paritairement à celui ou celle des consommateurs ou des usagers ;

- l'ouverture d'un statut coopératif qui, pour le capital, envisagerait un sort moins restrictif et moins ingrat que celui d'un dividende plafonné, et d'une résorption dans des plus-values collectives décidément impartageables. 
Soit, pour l'intergénération coopérative, trois problèmes de « communication »:

- entre les coopératives de services et les coopératives de travail

- entre coopérateurs de travail dans les coopératives de services

- entre le statut de travail et le statut du capital.

51 Du même coup, les principes (ou plutôt les pratiques) «de Rochdale » tenus pour ou prétendus tels s'en trouvaient « revisités ». Les « empires » d'un modèle dominant, celui des consommateurs associés et de leurs techno-structures, s'en trouvaient interpellés.. Et les deux statuts, celui du travail comme celui du capital, s'en trouvaient reconsidérés. Mais aussi, c'est que, en France comme en Angleterre, un courant avait préparé ces attestations manifestes dans une contestation latente: en France, un courant, dit " participationniste » ravitaillé par une tradition saint-simonienne ou fouriériste et leurs postérités; en Angleterre, un courant socio-chrétien nourri de nostalgie quarantehuitarde, et qui déjà, face aux Magasins de Gros (Wholesale), avait campé à Londres sa Cooperative Union et ses coopératives de producteurs... Dans le camps britannique, deux personnages en flèche et leurs facultés respectives : la mémoire de l'historien Hlyoake, l'imagination d'un leader, Vansittart Neale. Depuis 1885, les visites réciproques avaient été ininterrompues de congrès anglais à congrès français et réciproquement. En 1892 à Rochdak allait se tenir le vingt-quatrième congrès anglais. C'est alors, à l'occasion et en marge de ce congrès, que fut ourdie la concertation décisive, rédigé son proposai, et proclamée cette Alliance internationale des amis de la production coopérative, dont les péripéties et les postulats seront d'autant plus escamotés par l'historiographie subséquente que, dès 1895 , leur interprétation de l'archétype rochdalien, «selon Vansittart Neaule et ses amis ", passera à la trappe...

tout mériterait une restitution mouvementée. Elle a déjà été amorcée (cf. «Principes rochdaliens. Lesquels?», in AISC 10, 1961, P 3-38), y compris (p. 29-38) restitution bilingue du Proposai. Et elle méritera sa réitération puisque son triple profilage (cf. supra) s'avère à ce point survivant qu'il en ressuscite dans les lignes et entre les lignes préparatoire de Tokyo...

53 Comme déjà mentionné, les congrès de l'ACI ont particulièrement médité sur les significations culturelles de l'économie coopérative : ses horizons 2000 à Moscou (1980); ses finalités à Hambourg (1984); ses "valeurs» à Stockholm (1988). Et même, depuis Stockholm, une Commission indépendante a été chargée de donner une suite à cette axiologie en discernant ce qui concerne respectivement une éthique stratégique et une jurisprudence entrepreneuriale. C'était reprendre, sur de nouvelles donnes, la partie inaugurée et oubliée de 1892, comme les parties successivement jouées, en particulier dans les années trente et les années soixante. Y conviaient les métamorphoses de l'Ouest, les obsolescences de l'Est, les jeux et les enjeux du Nord-Sud, les revigorations de l'entreprise, les marginalisations des idéologies et les défis écologiques de la planète.

Préposé à l'animation de cette Commission indépendante, le Suédois Sven Ake Böök s'est évertué à lui faire valser «la valse des éthiques» et les premiers couplets de l'accompagnement tombent sur nos bureaux. Miracle ou paradoxe! Voici que trois couplets de 1892 remontent sur la scène, après avoir été, cent ans durant, rejetés dans les coulisses ou expédiés dans les praticables, et sans même avoir été soufflés depuis la niche d'un souffleur. Qu'on en juge sur citations ici traduites :

- Sur l'hégémonie des consommateurs et sa contestation: 
56 «Les Principes (actuels) ne sont pas assez universels. Dans leurs contextes et dans les origines ils sont trop marqués par les coopératives de consommation, et qui plus est européennes, et ils ne sont guère (not fully) applicables ni aux autres types de coopératives, ni au développement coopératif dans d'autre cultures »... Ou ailleurs, sinon réponse, du moins question: «Les organisations coopératives, celles des producteurs et celles des consommateurs, sont au service d'intérêts matériels opposés. Comme producteur, les membres "want to gel good payment for the investment and work», et comme consommateurs, "want to get good outcome of the incomes" (autrement dit, les premiers voudraient que soient mieux payé ce que les seconds voudraient payer moins cher...). « Il ne sera jamais possible d'harmoniser ces intérêts fondamentaux ». Ambitions (encore platoniques) : quelque chose comme un marché commun intercoopératif.

57 - Sur un nouveau statut des employés (producteurs ou travailleurs) dans les entreprises coopératives (de services)

58 C'est ce qu'avait demandé Albert Thomas, et dès les années vingt, sans pouvoir l'obtenir. $\mathrm{Et}$, il y a cent ans, ce fut le fer de lance de la pré-ACI (enterrée - avec fleurs et couronnes dès 1895). Mais voilà que cette clause est réitérée et réhabilité à la hauteur d'un principe ou d'une « valeur » :

59 «Le principe de Démocratie devrait être complété par une stipulation (a statement) sur la participation des employés dans la gestion démocratique des coopératives (...). En conséquence, les employés doivent occuper une place spécifique dans la démocratie coopérative participative. Entre autres, les coopératives devraient trouver des procédures (methods) correspondantes à celles de la participation aux bénéfices (profit-sharing) ou à des modèles de co-gestion (co-partnership), moyennant quoi les employés, à l'égal des usagers, obtiendraient un statut de sociétariat et de partenariat (ownership and membership) » etc, etc.

60 - Sur un nouveau statut du capital (et selon une lignée "participationniste " autrefois récusée mais maintenant revalorisée)

61 Selon les « anciens » principes (encore stipulés à Vienne en 1966) : la part du capital ne devrait être rémunérée, si elle l'est, que par un intérêt strictement limité... Interpellation : «il est devenu évident durant les dernières décennies qu'un tel principe inflige des limitations excessives à la formation du capital coopératif et à l'efficacité économique ». A la formulation "ancienne» est opposée une rédaction nouvelle: "S'agissant des parts de capital, de leur collecte et de leur gestion; les organisations coopératives devraient ajuster des procédures reposant autant que possible sur les contributions de leurs propres membres. Des parts de capital supplémentaires peuvent être collectées sous des formes qui soient compatibles avec la promotion des intérêts des membres, leur démocratie et leur indépendance. Les intérêts de ce capital devraient être "flexibles ", même s'ils sont limités à un niveau au-dessus du taux officiel »... Ou en plus bref : «Le principe de l'intérêt limité au capital devrait être considéré et formulé selon un mode plus flexible "...

62 NOVA ET VETERA. Les libellés de ces nouvelles propositions se trouvaient implicitement et explicitement dans ce cher vieux Proposai de juin 1892, il y a tout juste cent ans. Qu'on m'en croie ou qu'on s'en assure. Même si son texte en est à sortir du tombeau.

63 L'un des plus respectables exégètes du mouvement coopératif, W.P. Watkins, a écrit un jour : « On a dit justement que la coopération est un mouvement économique qui se sert 
de l'éducation, mais on peut tout aussi bien retourner la proposition et dire que la coopération est un mouvement éducatif qui se sert de l'action économique ».

Similairement ou inversement, on serait tenté d'écrire : «Si la communication devrait avoir pour fin un mode de coopération, la coopération devrait avoir pour moyen un mode, voire un régime de communication.

Communication entre ses populations composantes

Communication entre ses types d'entreprises, et en particulier coopératives de services et coopératives de travail

Communication entre ses appareils et ses réseaux, sa chaîne entrepreneuriale et sa trame associative, ses bases et ses sommets

Communication de subsidiarité entre ses ordonnances d'ONG et ses surordinations par l'OG, national, européen ou international

Communication de corrélations entre le tissu coopératif Nord-Nord et les terroirs coopératifs Nord-Sud

Communication entre l'économie coopérative ou intercoopérative et les économies sociales, leurs composantes associatives ou mutualistes, leurs interfaces avec l'économie collective, l'économie syndicale, l'économie participative, l'économie paritaire, l'économie locale, communale ou communautaire

71 Communication entre les créativités et les pérennités

72 Communication entre le mouvement économique qui se sert de l'éducation et le mouvement éducatif qui se sert de l'action économique

73 Communication entre les lignages macro-économiques déjà "arrivés » et les lignées micro-économiques encore arrivantes, l'économie «installée» et l'économie « souterraine », la formelle et l'informelle, l'« established » et la "grass roots », l'émergée et l'immergée, les situations « organiques » et les situations « critiques »

74 Communication entre la mémoire coopérative et ses souvenirs, d'une part, et, d'autre part son imagination et ses espérances

75 Communication entre ce qui fait courir des militances (cf. Yvon Bourdet) et ce qui fait durer des intendances

Communication entre ce qui offre les moyens de vivre et ce qui demande les raisons d'en vivre

Communication entre les solidarités fondées et les solitudes qui sont fondatrices... selon l'axiome de Charles Gide :

78 «Ce que nous savons, c'est qu'il n’y a pas une seule de ces sociétés (coopératives) parmi les 100000 qui existent dans le monde, il n'est est pas une seule qui eût vécu et duré si elle n'eut trouvé un homme qui vive pour elle, qui en ait été le fondateur et en reste l'animateur. Beaucoup plus nombreuses celles qui n'en ont point trouvé et qui, pour cela, sont mortes » (Economie Sociale, p. 190-191)

79 Et par conséquent, pour ce mouvement coopératif comme pour tout mouvement social: communication entre ce qui serait seulement le bonheur et ce qui serait solitairement une béatitude.

80 Sola Beatitudo. Beata solitudo. N'est-ce pas le paradoxe qui est aussi la clef de toute communication? 


\section{NOTES}

1. Généralement, c'est-à-dire sauf le cas des macro-sociétés encore Indemnes de ce processus de croissance, sauf également le cat d'une coopérative de production dont tous les travailleurs seraient sociétaire auxquels cas ledit quadrilatère se réduirait à un triangle.

2. Plusieurs autres versions ont complexifié la combinatoire entre ces quatre pôles. Qu'il suffise d'évoquer son algèbre selon que ces pôles se combinent à un, deux, trois ou quatre selon les $n+1$ cas de figure, et le type-idéal étant une communication quadripartite dans laquelle chacun des pôles communique non seulement avec chacun des trois autres mais aussi avec la variété de leurs tandems ou/et de leurs trios.

3. Certaines expériences de radio éducative où les radio-clubs sont placés eux-mêmes en situation émettrice permettent de supputer chances et risques d'un tel dispositif. Cf. par exemple Michel Bourgeois, Dissoo ou le malaise paysan au Sénégal (1966 1973). Essais d'analyse de l'évolution du monde rural sénégalais réalisée à travers les programmes de la radio éducative. Thèse de $3^{\mathrm{e}}$ cycle, Paris, $1975,498 \mathrm{p}$.

4. 4. Ch. Gide, L'Economie sociale, Jam. cit. p. 59

5. Cf. ASSCOD, 76, 1986 : Réunir l'entreprise coopérative ASSCOD, 83,1988: Vers une éthique coopérative

ASSCOD, 84-85, 1988 : l'avenir de l'entreprise participative et démocratique ... etc

\section{RÉSUMÉS}

À propos de la communication et des pratiques coopératives, l'auteur est amené à distinguer trois registres susceptibles d'attention :

- la communication au sein des organisations coopératives et les particularités de sa mise en œuvre étant donné les spécificités de leurs publics et le projet démocratique qu'elles incarnent.

- la communication inter-organisationnelle ou celle qui relie toutes les instances concernées par le projet coopératif.

- la communication qu'il nomme intergénérationnelle ou celle qui perpétue et renouvelle le discours axiologique qui fonde le projet coopératif.

\section{AUTEUR}

\section{HENRI DESROCHE}

«Son itinéraire passe par Économie et Humanisme et les communautés de travail dans les années quarante, la fondation du Collège Coopératif et des archives internationales de sociologie et la coopération dans les années cinquante, une direction d'études à l'École pratique des Hautes Études dans les années soixante, la promotion et l'animation de 
l'Université coopérative internationale dans les années soixante-dix/80. A publié une trentaine d'ouvrages dont plusieurs sont des classiques de l'économie sociale ». 